\title{
TapeBlocks: A Making Toolkit for People Living with Intellectual Disabilities
}

\author{
Kirsten Ellis \\ Monash University \\ Melbourne, Australia \\ kirsten.ellis@monash.edu
}

\author{
Emily Dao \\ Action Lab, Monash University \\ Melbourne, Australia \\ mdao1@student.monash.edu
}

\author{
Osian Smith \\ Swansea University \\ Swansea, Wales \\ o.l.smith@swansea.ac.uk
}

\begin{abstract}
Stephen Lindsay
Swansea University

Swansea, Wales

s.c.lindsay@swansea.ac.uk

ABSTRACT

The accessibility and affordability of tangible electronic toolkits are significant barriers to their uptake by people with disabilities. We present the design and evaluation of TapeBlocks, a low-cost, low-fidelity toolkit intended to be accessible for people with intellectual disabilities while promoting creativity and engagement. We evaluated TapeBlocks by interviewing makers, special educational needs teachers and support coaches. Analysis of these interviews informed the design of a series of maker workshops using TapeBlocks with young adults living with intellectual disabilities, led by support coaches with support from the research team. Participants were able to engage with TapeBlocks and making, eventually building their own TapeBlocks to make personal creations. Our evaluation reveals how TapeBlocks supports accessible making and playful discovery of electronics for people living with disabilities, and addresses a gap in existing toolkits by being tinkerable, affordable and having a low threshold for engagement.
\end{abstract}

\author{
Patrick Olivier \\ Action Lab, Monash University \\ Melbourne, Australia \\ patrick.olivier@monash.edu
}

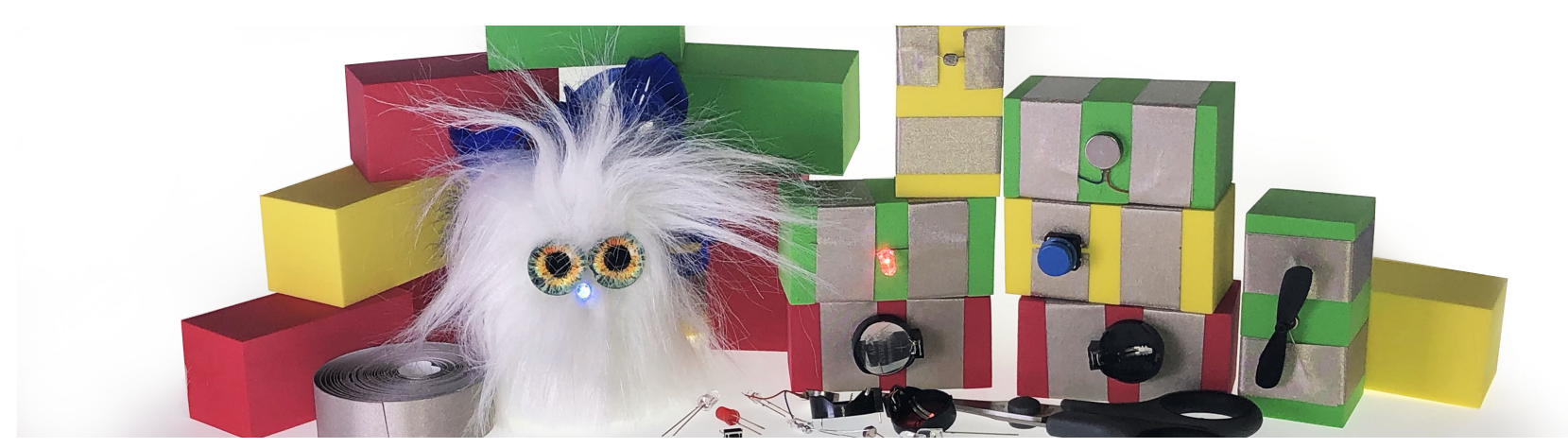

Figure 1: TapeBlocks: stacks of blocks, a character, materials and components.

This work is licensed under a Creative Commons Attribution International 4.0 License.

CHI '21, May 8-13, 2021, Yokohama, Japan

(C) 2021 Copyright held by the owner/author(s)

ACM ISBN 978-1-4503-8096-6/21/05.

https://doi.org/10.1145/3411764.3445647

\section{CCS CONCEPTS}

- Human-centered computing $\rightarrow$ Accessibility technologies.

\section{KEYWORDS}

Making, intellectual disability, tangibles, accessibility

\section{ACM Reference Format:}

Kirsten Ellis, Emily Dao, Osian Smith, Stephen Lindsay, and Patrick Olivier. 2021. TapeBlocks: A Making Toolkit for People Living with Intellectual Disabilities. In CHI Conference on Human Factors in Computing Systems (CHI '21), May 8-13, 2021, Yokohama, Japan. ACM, New York, NY, USA, 12 pages. https://doi.org/10.1145/3411764.3445647

\section{INTRODUCTION}

Building physical electronic circuits is perceived as complex and intimidating for novice users [1]. As a result, a number of tools and electronics engagement kits, grounded in the maker movement, have been designed to encourage curiosity and exploration for novices. Moreover, there is an established research agenda that seeks to simplify and expand the traditional methods and audience for electronics knowledge acquisition and making participation, and the resulting range of diverse kits for tangible electronics has genuinely improved access $[18,21]$. However, for many people with intellectual and/or physical disabilities, existing makerspaces and engagement kits do not overcome all of the accessibility and affordability barriers [26, 34, 35]. 
The accessibility of electronic maker kits can be a barrier for people with disabilities $[5,22,32]$ due to the cognitive load associated with remembering electronics concepts or the physical motor skills [14] required to engage in making. Beyond this, people's perceptions of themselves might not include the ability to engage with electronics activities reducing motivation to study them. However, designing accessible electronic maker kits leads to a number of trade-offs. Visibility of circuit structure is important to understanding electronics but accessible kits hide or abstract away from these complexities to make them simple to use $[9,17,18]$. This reduces the educational impact in favour of lowering barriers to entry. This is seen in accessible making more generally where trade-offs are made that hide structural complexity but sacrifice learning [17, 21].

Our response has been to create TapeBlocks, a toolkit for building circuits that can act as a first point of contact with making for people living with a range of disabilities. We designed the toolkit, with a focus on its physical attributes, to minimise trade-offs and access barriers. In doing so, we also challenge people's perceptions of how circuits should be constructed and by whom, regardless of their physical abilities. TapeBlocks (Figure 1) are colourful foam blocks that use conductive tape to connect electronic components as well as to make positive and negative electrical connections between the blocks. We design TapeBlocks using a set of qualities derived from literature on accessibility, electronics toolkits $[18,21,26]$ and makerspace accessibility as well as extensive experience from the researchers' work with adults with disabilities. The work is part of an ongoing endeavour to include people with a range of disabilities in making. TapeBlocks are accessible because of their chunky physical form and the simplicity of the connections. They encourage tinkerability as they are flexible, robust and unintimidating. Finally, they are affordable because they are made from individual components which can be reused.

In this paper, we propose a range of components that can be used to create different typese of TapeBlocks including power, input and output options that can be combined to make a variety of working circuits. We undertake a series of evaluation sessions with makers, special educational needs teachers and support coaches. Analysis of these sessions informed the structure of a series of workshops with young adults living with intellectual disabilities which ran over two weeks led by support coaches with some support from the research team. We leverage the qualities of TapeBlocks to encourage people to play and experiment before they progress to making their own set of TapeBlocks to develop their making skills and start to express their own creativity. We found that the workshop participants were able to engage with TapeBlocks and meta-making, building their own personal creations at the end of the workshop.

We make two significant contributions in this paper: 1) we introduce TapeBlocks, an accessible toolkit designed to address issues in makerspace access and electronics education for people living with a range of disabilities and 2) we present the results of the design and evaluation of TapeBlocks in collaboration with makers, special education needs teaching staff, and young adults living with intellectual disabilities.

\section{RELATED WORK}

Maker culture is a global phenomenon that promotes and supports people engaging in diverse activities, ranging from software hacking, traditional crafts, and tinkering to digital fabrication such as $3 \mathrm{D}$ printing, as a way to design and build typically for one's own fulfillment, rather than for commercial benefit [34]. The recent increase in the availability of digital manufacturing tools, such as laser cutters and 3D printers, has expanded the range of people engaged in maker culture and resulted in making activities growing in popularity [3]. Here, we discuss prior work in the areas of engaging people in maker culture, current provision of access to makerspaces and examples of accessible toolkits.

In this paper we use the term intellectual disability as this reflects the terminology used in the country the work is performed in and the care organisation we worked with. All our workshop participants had diagnosed intellectual disabilities. Intellectual disabilities are defined in Australia in cases where a person shows "difficulty learning or understanding things" for longer than 6 months [12] and who often have other physical coordination problems. The design is informed by literature and experiences with people with traumatic brain injury which causes various cognitive issues including memory loss, aphasia and problems with long term attention [28] , as well as work with people with limited fine motor skills. We envision TapeBlocks being more widely useful because of the consideration for a variety of cognitive challenges people can face and because of their focus on supporting people with limited manual dexterity.

\section{Benefits of Engaging People in Maker Culture}

Hurst and Tobias [19] suggest that in addition to encouraging a sense of creativity, maker culture enables independence and supports wellbeing amongst its members, makers. For many makers, the primary focus is not the creations themselves ('what' was made), but rather the act of making, ('how' it was made) [34]. For these people, the benefit of maker culture lies in providing an environment that empowers them to be creative and expressive. For example, Güldenpfennig [16] notes that in addition to providing a means for creative exploration and learning new skills, making also allows makers to create meaningful and personal presents to give people they care about. Makers derive satisfaction from the ability to make bespoke, personal creations [13, 19, 29, 36].

People living with disabilities often need to modify technology to make it accessible and research has demonstrated that Do-ItYourself (DIY) assistive technologies and modifications to existing commercial solutions provides a way to address the limitations of one-design-meets-all products [19, 29]. In recent years, HCI has also taken an increased interest in the role making can play in wellbeing [15, 19, 34]. O'Kane et al. [29] outlines emerging trends where people want to customise the design of their own DIY health technologies, as seen in the \#WeAreNotWaiting movement in the diabetes community [29]. In addition, the work of Vincent et al. [36] shows how adults living with Type 1 Diabetes are adopting and using devices in a variety of ways to suit their individualised daily needs.

\section{Current Accessibility Of Makerspaces}

Despite having a range of benefits for their wellbeing, making has significant access barriers for people living with disabilities. Makerspaces rhetoric is inclusionary, utopian, and techno-solutionist, but in reality, as with many hobbies, there are issues with access 
relating to gender, socio-economic status and age [34]. Research carried out by the MakerMedia magazine at one hackathon found that $97 \%$ of adult attendees had a college degree, $70 \%$ had a graduate degree, and the average income was of $\$ 130,000$ per annum [24]. Affordability is a significant barrier to access. People with disabilities often experience financial disadvantages compared to their peers [33].

For example, within the UK, people with disabilities are significantly less likely to be employed and when employed, will earn on average $18.6 \%$ less than their non-disabled colleagues in the same job [33]. Reviews of makerspaces by Taylor et al. find that while makerspaces want to reach out to a broader range of people, many of them are unable to, citing challenges around limited resources and lack of training on how to encourage or support makers with disabilities [34].

Furthermore, despite the growing availability of open-source designs including many for people with disabilities, Buehler et al. [10] noted the rarity of that DIY-AT being created by people with disabilities. Barriers arising from the lack of adaptation to include people with different physical abilities also makes people with disabilities feel culturally excluded from maker practices. Existing work on introductory toolkits for making has primarily focused on an ablebodied population with good vision and fine motor skills $[4,9,30]$. This creates significant barriers to entry for people with disabilities, specifically those with intellectual disabilities, as underlying health condition often result in limited dexterity [14] and most of the components of these toolkits are small and difficult to manipulate. Furthermore, existing work on accessible technology design focuses on those with motor, hearing or visual impairments [27] and Chapkol et al. [11] highlights a need for the development of applications that carefully consider the needs of the neurodiverse.

Toombs et al. [35] also highlight that many people feel intimidated by the idea of getting involved in 'hacking'. This can be due to perceptions of makerspaces as high tech environments involving complex and novel tools. In addition, maker culture relies on an ability to do independent research to learn about new tools and, although this skill is one of the most useful ones it develops, it creates another barrier as the skill is not explicitly taught or widely known [35]. This leads to a culture that is often described as "sink-or-swim" resulting in many members needing "onboarding" and support before then can start the making process but not being able to consistently access it. This in turn results in minorities and women feeling lost in the making community, and seeing others hacking alone, discourages many people from engaging [6]. Lewis [23] noted that many women feel intimidated due to the psychological barriers and limited participation by women in makerspaces. Potential participants can also be deterred by the feeling that making is a social clique, and they feel that they have very little in common with current makers.

\section{Designing Accessible Toolkits}

Despite the aforementioned barriers relating to makerspaces accessibility, several researchers have explored making electronic and maker toolkits more accessible [2, 9, 18, 26]. Established makers have shared assistive technology at maker events or hackathons and on digital platforms such as Thingiverse focusing on health and wellbeing [10]. Buehler et al. [10] explored barriers to making $3 \mathrm{D}$ printing more accessible in special education settings and Barbareschi et al. [2] developed Tip-Toy, an electronic toolkit with physical blocks to teach basic computing concepts through music for children with different visual abilities. Using Tip-Toy, participants could recognise blocks either touch or sight and outputted music, allowing learners with reduced visual abilities to participate in accessible and enjoyable programming experiences. Further, Hollinworth et al. [18] extended littleBits, which are printed circuits with connected electronic components, by enlarging them to introduce electronics to children with learning difficulties. Preliminary evaluation showed that users found the modifications made to littleBits made them easier to handle and manipulate [18]. The kits are prefabricated and the tight focus supports people by reducing cognitive overhead, however, this configuration of the toolkit also makes exploratory or creative making harder.

The concept of using blocks as a method of making for individuals is not limited to the physical environment $[8,26]$. Many block-based programming languages, such as MIT Scratch [25], have become popular over the last few years, but rely on visual metaphors and traditional gestures such as drag and drop which are not accessible to those with visual impairments. Milne et al. [26] developed Blocks4All, a block-based programming language with VoiceOver for primary aged children with visual impairments. Their work identified important design considerations for accessibility, including a focus on simplicity and reducing the number of focusable items; conveying information in different ways and indicating error states, for example, if a block cannot be placed.

Adding to Milne et al.'s design considerations [26], Leduc-Mills et al. [21] suggests that the designers of construction kits for children with intellectual difficulties should design around the following architectural principles: (1) low floors with ramps, to ensure that kits are straightforward for children without special needs but can be made more accessible for those with disabilities; (2) high ceiling with tall ladders, by allowing more complex projects to be developed with ladders to help those with learning difficulties to reach their full potential; (3) wide wall and frames of interest, to allow children to explore their expressions, to also guide those with ASD (autism spectrum disorder) to focus on more repetitive tasks with a specific set of tools, actions and characters; and (4) reinforced corners, as to offer more support when required [21].

We ground our design of TapeBlocks in these accessibility principles, and contribute to the ongoing body of work on accessible toolkits for the neurodiverse.

\section{DESIGN RATIONALE}

Our research is motivated by our (the authors) collective experiences of running making workshops with people with varying abilities, including people who have an intellectual disability, parents of children who have a disability, blind and low vision adults, and adults living with traumatic brain injury. Not only have we found that makerspaces and making equipment are almost universally inaccessible, researchers and practitioners face numerous challenges when seeking to involve people with disabilities in making activities, including ethical assessments, safety risks and practical challenges of bringing them into those spaces. Furthermore, 


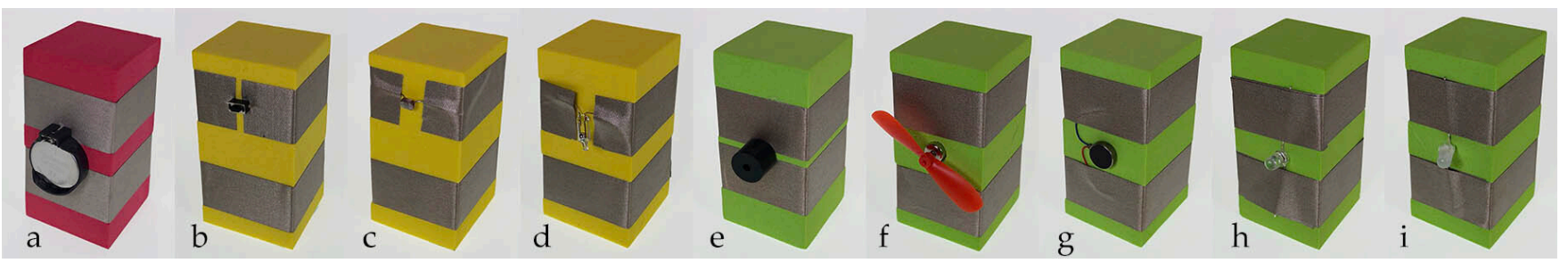

Figure 2: Types of TapeBlocks: a) power, b) button, c) light dependent resistor, d) tilt switch, e) buzzer, f) fan, g) vibration motor, h) Red, green and blue (RGB) colour change and i) bicolour LEDs

existing toolkits, ranging from electronics through breadboarding or soldering $[18,20]$ to sewing kits, largely assume capabilities such as fine motor skills and high visual acuity.

The requirements for a maker toolkit for people with intellectual disabilities range from physical and visual characteristics of the artefacts themselves, to the design of the making process for which the toolkit is intended. Making activities have varying complexity, and the process of making can often be long and involve many trial and error cycles. The try, fail, adapt and retry rhythm of making is not achievable for a person living with an intellectual disability using conventional maker toolkits. Drawing on prior work on accessibility and toolkits and our experiences as both researchers and practitioners, our goal is to create an introductory toolkit that does not require fine motor skills, is inexpensive and reusable, and affords an authentic making experience of achievable length.

\subsection{Introducing TapeBlocks}

TapeBlocks are foam building blocks that use conductive tape rather than wires to form a circuit. There are three classes of TapeBlocks that can be combined to create circuits: power, sensor and output (Figure 2). The blocks themselves are chunky $(7 \mathrm{~cm} \times 3 \mathrm{~cm} \times 3 \mathrm{~cm})$ children's EVA foam building blocks. TapeBlocks are constructed using electrical components such as Light Emitting Diodes (LED)s, resistors and vibration motors. The conductive tape which holds the components on the blocks is a flexible polyester cloth tape that has a conductive copper and nickel surface, the adhesive is conductive acrylic meaning that the top or the underside of the tape can be used to form connections. The conductive tape ( $2 \mathrm{~cm}$ wide) provides a large area that can be used to make connections between the blocks. Two parallel strips of conductive tape are wrapped the entire way around the short side of the blocks so that they can be connected with another block along the long side. For a power block, one of the strips is connected to the positive terminal on the power source and functionally becomes the positive wire, while the other acts as the negative wire. A second block with a component is required to make a circuit. Each component is placed either under or on top of the conductive tape in order to bridge the positive and negative lines (Figure 3). When a power block is connected with the correct polarity to the component block, touching the strips of tape creates a circuit and the component activates.

The minimum tools required to make a TapeBlocks circuit consist of (1) a power source (i.e., a battery) and (2) a component (i.e., a light source). By creating these on two separate blocks, moving them together and apart creates a switch. The TapeBlocks methods of inserting components under and over the conductive tape makes it possible to create a range of blocks including power blocks, button blocks, light sensors, tilt sensors, buzzers, fans, vibration motors, RGB colour change LEDs, and a bicolour or non polar LED (Figure 2). This in turn enables a variety of cause and effect interactive experiences and provide visual, tactile and audible feedback.

\subsection{Aspirations for TapeBlocks}

We formed a set of qualities that we felt were desirable for an accessible electronics toolkit. When developing TapeBlocks, there were a multitude of design choices regarding configurations and materials. We followed an iterative process and refined TapeBlocks to be an accessible toolkit with the following characteristics.

3.2.1 Tinkerability. TapeBlocks are designed for tinkerability [31], they aim to be flexible and easily reconfigured for other purposes. The choice of colourful EVA foam is intended to make TapeBlocks seem familiar, friendly, soft and yet robust. We experimented with other materials and found the EVA foam blocks afforded building, stacking and combining. Pre-made blocks make it simple and intuitive to make a circuit and aligns with Leduc-Mills et al.'s principle of low floors with ramps [21]. Additionally, a variety of electronic components, including those not discussed in the paper, can be explored using TapeBlocks. TapeBlocks allow multiple components to be combined into a single block or alternatively several blocks can be wrapped in conductive tape and combined with component to create unique pieces (Figure 7). They can be used to create objects that are of personal interest to users, such as cars, trains or animals.

Affordability is an important facilitator of tinkerability as creation and failure is more acceptable if the cost of failure is not prohibitive. Because TapeBlocks are made from individual components and not specialised components, the cost of building and using them is low. An integrated TapeBlocks circuit with a block, battery, battery carrier, LED and tape can be obtained for under USD $\$ 1$ and the components required for the 9 blocks in figure 2 can be purchased using bulk supplies for USD\$12. As there are no specialist components, all of the components can be purchased locally from the cheapest source. The only unusual material is the fabric conductive tape which can be ordered through online distributors. Within the context of our participants, this is more affordable than the available alternatives.

With regard to robustness and re-usability, the TapeBlocks making activity can only be undertaken once, as the tape cannot be 


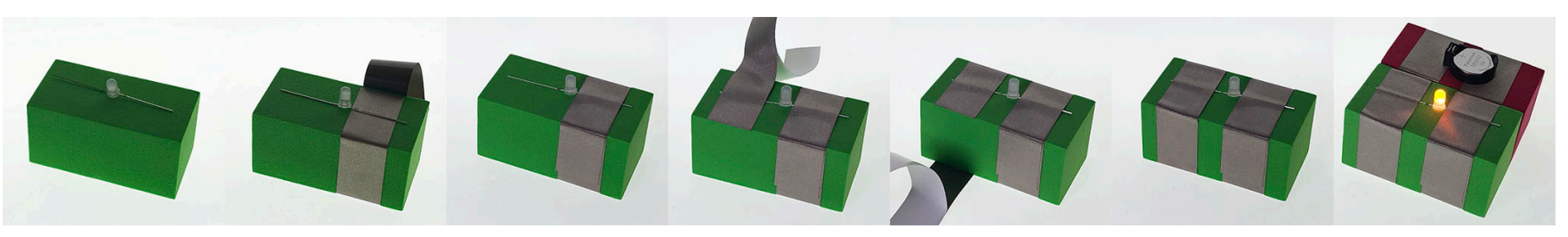

Figure 3: Making an LED TapeBlocks by inserting the LED under the conductive tape

removed after being placed on the blocks. However, once the TapeBlocks have been made, they can be re-used multiple times. If the components become too dirty, they would eventually need to be replaced, however we note that we have used and demonstrated some of our test TapeBlocks over a thousand times and have not come across this issue. The tape used for TapeBlocks maintains its conductivity as it does not tarnish or tear, compared to its other tapes such as copper tape. The batteries need to be replaced, however it is possible to make TapeBlocks with other power options. These include but are not limited to: 5V USB rechargeable power packs, solar panels, which were not explored in this paper.

Beyond this, the ability to reconfigure the tools themselves adds another layer of complexity and self expression. This activity is necessarily more complex than connecting the blocks together and fits with the high ceiling and ladders paradigm for toolkits [21].

3.2.2 Low threshold for engagement. We intend for TapeBlocks to require a very low threshold for engagement. For users without fine dexterity, TapeBlocks should offer a low physical threshold, such that if the blocks are put on a flat surface they are able to be pushed together with the back of their hand to form a working circuit. The EVA foam blocks are large enough to easily physically manipulate and have no sharp edges. The use of conductive tape rather than other common alternatives such as breadboards, soldering, sewing created a much lower barrier to entry. The physical making of the blocks also tries to use tools and materials that are likely to be simple, and familiar and easy to use.

We also aim for TapeBlocks to be simple and intuitive without the need for complex instructions. To create a circuit, all that is required is to push a power block and an LED block together. The connection areas for the circuit are large, which is intended to encourage participants to work out how to make the circuit with TapeBlocks or discover how to create a circuit through exploration of the potential combinations which are constrained and error tolerant.

3.2.3 Meaningful making and social character. TapeBlocks let people living with disabilities actively participate in the maker community by providing an activity that was previously inaccessible to them. By including people with disabilities in these activities, our hope is that we provide them with opportunities to create a sense of social connection and discuss what they are creating. Participating in making as a group can provide an environment where concepts and language can be practiced and names can be given to new ideas and concepts. Having multiple sessions of making TapeBlocks may offer opportunities for consistent reinforcement of the new skills and concepts that are being acquired.
The acquisition of new skills is exciting and electronics are often perceived as a challenging knowledge domain. Through the use of TapeBlocks as a toolkit that supports the building of knowledge about electronics, a challenge is met in a field that is often considered beyond a person's ability. This can lead to a personaly meaningful experience and a sense of pride at completing a challenging task. Finally, personal meaning may also come from creating objects that take effort and are of interest to the maker. The creations can be decorative or useful and are often shared with others and are kept as a reminder of the experience.

\section{METHODOLOGY}

The concept behind TapeBlocks emerged over multiple different projects working with people with a range of intellectual disabilities, making it challenging to point to a single formal design method we used. However, once the idea was in place, we evaluate TapeBlocks using a two-phase mixed-methods approach to understand the role that they can play in engaging people living with intellectual disabilities in making. The first phase focuses on understanding makers and special education professionals' perspectives on TapeBlocks, how they can be integrated into their practice, pragmatic concerns about their deployment and whether there are any ethical issues associated. In the second phase, we further refine and evaluate them through remote maker workshops, conducted with young adults living with intellectual disabilities and led by teachers and support coaches who took part in phase one. These workshops allow us to see how well the TapeBlocks activities are received, identify specific issues with their design, check that TapeBlocks workshops can be delivered by coaches and teachers without electronics background, and gauge the extent to which they engage participants with intellectual disabilities.

\subsection{Design with Makers and Coaches}

In the first phase we conduct one-on-one interviews with makers, and participatory design sessions with professional support coaches and teachers at an Non-Government Organisation dedicated to working with young adults with intellectual disabilities.

We visited three makers in their makerspaces for one-on-one interviews to understand their perspectives on bringing people living with intellectual disabilities into makerspaces. The makers discovered making through their study of Computer Science, their work as an academic or as a professional programmer. We introduced TapeBlocks to the makers at the end of the interviews and, after letting them play with them, talked about the opportunities they saw for TapeBlocks. The interviews lasted roughly one hour each. 


\begin{tabular}{|c|c|}
\hline Type of participants & Number of participants \\
\hline Makers & 3 \\
Support coaches & 3 \\
Teachers & 1 \\
\hline
\end{tabular}

Table 1: Overview of makers and special education professionals in phase one

Additionally, we held participatory design sessions with three support coaches and one teacher. These sessions had to be conducted remotely due to COVID-19 restrictions, and TapeBlocks kits were sent to them by postage prior to the sessions. We followed a participatory design approach using group sessions to evaluate TapeBlocks design and create a structure for remote workshops for the NGO's clients. The coaches and teacher all worked in a professional capacity with young adults with intellectual disabilities and regularly led lessons for them in person. At the start of the process, they already had some limited experience running remote classes. We wanted to gain insights from them about the barriers and enablers to making activities for adults with intellectual disabilities. Additionally, we also wanted to understand the practicalities of using TapeBlocks in a remote setting. We conducted three sessions each lasting approximately two hours with all four of them present along with two researchers. The first two sessions were conducted on Zoom and involved completing an online collaboration board (Figure 4). We held the last session in LearnCube (Figure 5), the educational software the NGO use to deliver their lessons, as the workshops with people with intellectual disabilities would be delivered through the same software and we wanted to familiarise ourselves with it.

\subsection{Makers and Coaches Sessions Outcomes}

The material from the transcripts, Miro boards, and workshops recordings were analysed using thematic analysis following the method outlined in [7] to create a simple, structured understanding of the opportunities and challenges that TapeBlocks and online learning would present. This analysis was presented back to the support coaches as part of our effort to create a shared understanding of how we should structure TapeBlocks workshops for remote delivery with their clients.

The coaches and makers all liked the TapeBlocks as they were presented to them. They quickly appreciated some of TapeBlocks' qualities, such as their soft foam construction, bright colours and easy configuration, maker 2 said "I think like they're nice tangibles, their squidginess encourages you to snap them together and try kind of silly things and see what works." The makers and support coaches suggested that the blocks follow a colour coded traffic light system, an idea that the research team had considered but rejected due to a few seasons: (1) the flexibility on choices of colour are perceived as an advantage, (2) current standards in electronics do not follow the traffic light system, and (3) this would put an additional constraint on participants to learn something else. However, due of the strong support, we changed the introductory kits to follow a traffic light red-yellow-green system (Figure 6,C). All of the coaches and makers enjoyed playing with the blocks and built their own creations from TapeBlocks. When thinking about the role that TapeBlocks could play in engaging people living with disabilities in making and electronics, their ideas shared three common themes.

4.2.1 Theme 1: Playfulness and engagement. All makers and support coaches emphasised that engaging the participants in the workshops would be central to success in different ways. Makers highlighted that personal interest in their creations was a significant factor in creating and maintaining engagement throughout the life of a project. One maker suggested that we ask participants to think about what "would be cool" or "be better than what [they] have seen" while another remarked that often engaging projects are those that are "very personal and very specific to that one person." Coaches stressed the importance of engagement with the concept. Coaches suggested that we focus on making TapeBlocks workshops that deeply engaged a small number of their clients and avoided sacrificing engagement for wider participation by, for example, making the workshops too simple. Coaches felt that their challenge, in general, lay in finding deeply engaging workshops that motivated clients to push themselves to learn a new skill rather than the figuring out their approach for workshop delivery. When thinking about how to make TapeBlocks more engaging, the coaches suggested that more actuators that produced movements would help. The makers thought because of how the blocks looked they would benefit from having an example pre-made "I would also probably, depending on the cohort, just because it looks quite unassuming, I would probably actually come in with something built with them already just as an example, just to give them an idea." However, the coaches planned to use the ambiguity of the blocks in their lessons by having clients initially explore the blocks without guidance to try and work out what they might be used for.

4.2.2 Theme 2: Learning and challenges. Makers in particular emphasised that trial and error was a vital part of the educational process in making and that, to teach this, TapeBlocks needed to be able to push people outside of their comfort zone. One maker said, "By intentionally making it more prone to failure, I actually think there's a lot to be learned from that". Coaches also thought that this was important, but framed their discussion of challenge in terms of the intellectual disabilities of their clients. They noted that TapeBlocks would be "fine for low and mid-dependency groups" but they doubted that they would see use with their high-dependency groups. Despite this, they were quick to point out that they did not think this meant the barrier for entry should be lowered more. There was some clear divergence here as some makers thought "for somebody who had impairment in their motor skills, you would probably have to have the blocks already made" but the coaches did not agree with this.

4.2.3 Theme 3: Lesson planning. Makers thoughts about the structuring of TapeBlocks workshops centred on embedding the curriculum within the tool "ideally the tool would tell you or would at least help you or provide some kind of scaffolding for you to figure it out". This is a central part of maker culture but the approach did not resonate with the coaches who focused more on the specifics of planning out lessons with the TapeBlocks. Our NGO collaborators were more concerned about understanding how they could teach their participants to use the TapeBlocks and how it could be fit into their existing practices. We found that workshops were typically grouped into portfolios of work that were run once or 


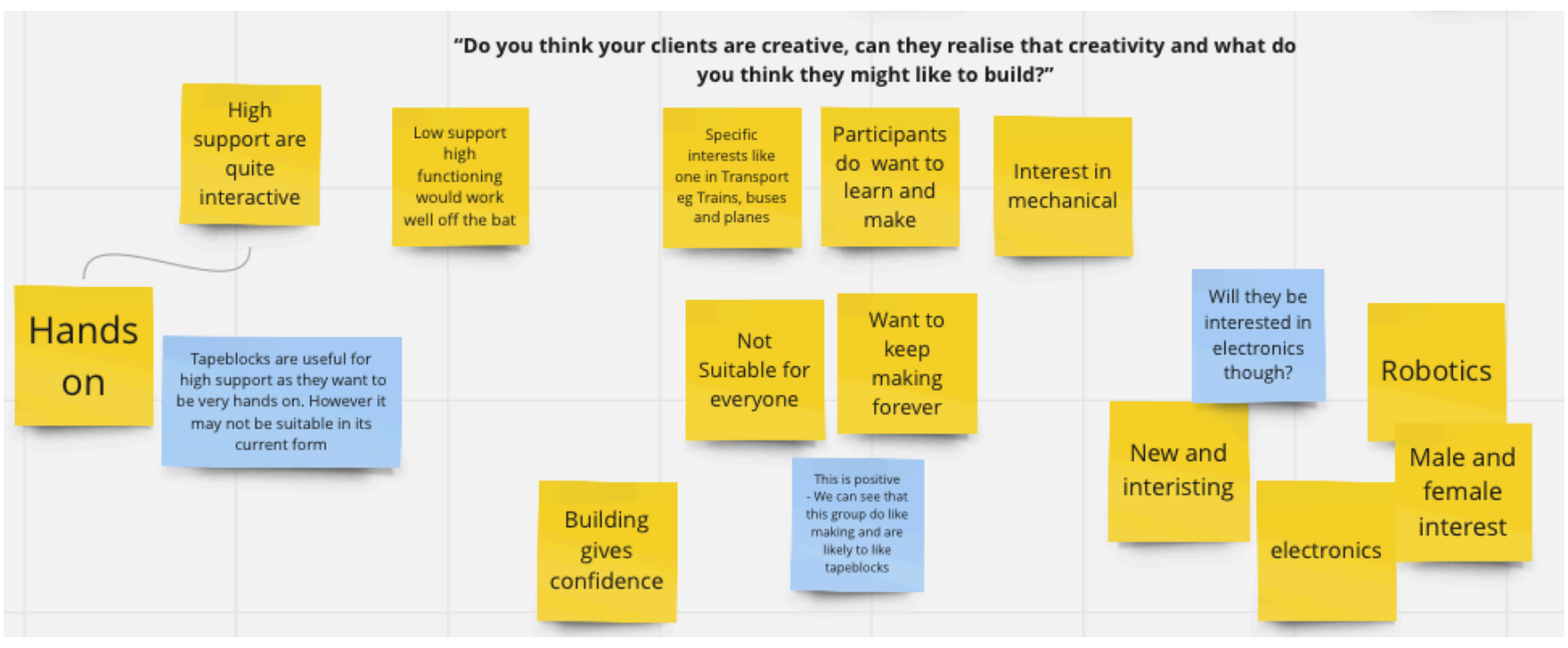

Figure 4: Miro Board with yellow and blue sticky notes

twice a week for two to six weeks. In our second and third sessions with the coaches, we helped develop a set of skills evaluation forms that they used to structure other work (Table 2) and a short lesson plan. Our aim to evaluate the TapeBlocks aligned well with their practices around portfolio review.

\subsection{Remote TapeBlocks Workshops}

The second phase of our evaluation consisted of observing and helping to run four remote, two-hour long maker workshops led by the support coaches. The workshops took place over the course of two weeks for four young adults with intellectual disabilities in the LearnCube environment (Figure 5). Participants had the TapeBlocks kits posted to them prior to the workshops and the coaches had the sets they used in phase one.

The workshops followed the agreed structure from phase one:

- Workshop one focused on introducing the basic functionality of TapeBlocks building up from the simplest combination

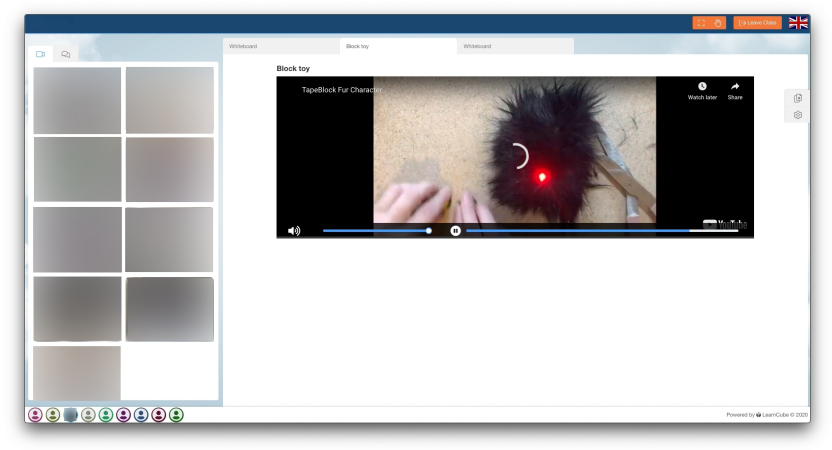

Figure 5: LearnCube environment with participant videos on the left possible of one power block and one LED to combining power, a sensor unit and an actuator. For example, making a noise come on when the TapeBlock is placed in the dark with a battery, beeper and a light dependent resistor block.

- Workshop two introduced the idea of building simple TapeBlocks for yourself using conductive tape, pre-cut EVA foam and electronic components. For example, participants would measure and cut conductive tape, find a battery and battery holder, and assemble them together before combining them with an LED block they have also built.

- Workshop three let participant works on creating the more complex sensor blocks introducing the idea of breaking and closing a circuit depending on a variety of detectable events. Here the form of the conductive tape on the TapeBlock is more complex and so the workshop is more challenging than workshop two but reinforces the same basic skills.

- Workshop four gave participants a chance to construct TapeBlocks based on a single foam block, completing a basic electronic circuit and allowing them to add multiple sensors and actuators as desired. The TapeBlocks is wrapped in materials of the participant's choice and they create a simple toy out at the end of the process (Figure 7).

To understand how these workshops would work without the presence of experienced makers, they were led by the support coaches who had participated in phase one. Each learning coach was supporting two to three participants for all sessions. At times, some of the participants received in-person support from caregivers, but not consistently. Researchers were on hand to offer help if it was needed because the research team members did not feel it was appropriate to let participants fail with the TapeBlocks because of oversights in our design and because the coaches felt some worry about delivering the TapeBlocks material alone on their first attempts. 


\begin{tabular}{|l|l|l|l|l|}
\hline $\begin{array}{l}\text { During the demonstration } \\
\text { of TapeBlocks }\end{array}$ & Independent & With support & $\begin{array}{l}\text { With prompting } \\
\text { and support }\end{array}$ & Not yet achieved \\
\hline $\begin{array}{l}\text { Can identify a battery } \\
\text { TapeBlock }\end{array}$ & & & \\
\hline $\begin{array}{l}\text { Can identify a LED } \\
\text { or motor TapeBlock }\end{array}$ & & & \\
\hline $\begin{array}{l}\text { Can identify a sensor } \\
\text { TapeBlock }\end{array}$ & & & & \\
\hline
\end{tabular}

Table 2: An example of a participant's portfolio review for Workshop 1

\subsection{Remote Workshops Outcomes}

The four workshops were recorded and then reviewed by members of the research team. The LearnCube environment presented some challenges in observing participants as video feeds are small (Figure 5 ) and audio and video would cut out for several minutes at a time.

4.4.1 Workshop One. The initial workshop with participants saw them being encouraged by the coaches to start by "playing around" with the TapeBlocks, exploring how they could be used and working out the steps needed to insert batteries into the holders. Participants had working two-block creations within around 15 minutes with encouragement but no teaching from the coaches despite the batteries presenting some challenges, with some participants inserting them the wrong way around. The sensor blocks were more challenging to understand, one of the coaches offered "You get a million points if you can work out what the [Light Dependant Resistor] does" but participants were happy when they were able to start using some of the more complex actuators and sensors. The participants enjoyed exploring the diversity of sensors and actuators but some of them struggled to hold sets of three blocks together as the EVA foam could spring apart. The camera link was not ideal when offering support, as coaches could not always tell if a problem was being caused by a broken block or the participant simply not holding them together firmly enough. The traffic light colour coding of the TapeBlocks that the coaches and makers suggested (Figure 6, C) compensated for the poor camera by helping the coaches guide participants to the right block as they could hold up one yellow block after another to their webcams to be told when they had found the right one. The skill of the coaches was clearly important in this phase as they timed moving from unstructured exploration of the TapeBlocks to more systematised work carefully.

4.4.2 Workshop Two. The second workshop started with a 15 minute recap and the fourth participant joined the workshop for the first time. They were able to catch up with the other participants

\begin{tabular}{|c|c|c|}
\hline Participant ID & Age & Gender \\
\hline 1 & 29 & Female \\
2 & 20 & Male \\
3 & 27 & Female \\
4 & 19 & Male \\
\hline
\end{tabular}

Table 3: Overview of participants in phase two with some help from their parent who was also on the call with them. The process of making red and green blocks started well with participants familiarity with the blocks lending them a sense of confidence working with the new blocks. Participants faced some difficulties making the blocks with tape too far apart to be bridged by a component and driving battery pins through tape and into the EVA foam. They found the tape more fidgety to work with than the foam blocks, leading to difficulties with cutting it to length. Finally, some of the components were confused by participants and coaches, for example, the difference between a tilt sensor and an LED was not obvious to most participants. However, the challenges were all overcome by participants and some of them even started to work ahead of the group making different green TapeBlocks.

4.4.3 Workshop Three. The third workshop focused on building yellow sensor TapeBlocks. Here the patterning of the tape on the TapeBlocks was an issue for participants as the sensor blocks need a break in the tape on the rear face of the block (Figure 6, A) and conveying this to participants was difficult. The main issue came when participants tried to cut the tape to a length that would let them wrap it around the block while leaving a gap at the back. Coaches struggled to find a way to articulate how to cut the tape to length, trying out instructions such as making it the length of one and a half faces of the block. However, participants were confused about which faces the coaches meant and how to position the tape. Fortunately, having had the chance to see this issue in the previous workshop, we had prepared a video to act as supporting material that outlined how to measure and cut the conductive tape in this case and one of the research team stepped in to go over this process live with the participants. Participants needed time to work through their problems and it took up to 40 minutes for them to understand what was going wrong but they had audible "ah-ha!" moments when they understood the necessary layout.

4.4.4 Workshop Four. The final workshop focused on participants building their own personal creation, a toy like single integrated block with light up nose (Figure 7). We anticipated this being particularly difficult but we had a member of the research team take part in the workshop to give a step by step guide. One participant was very adept at this stage and actually started to walk the others through their process as they worked step by step while thinking aloud. However, another participant became disengaged while support was focused on other students. This workshop highlighted some of the limitations of the teaching environment for this style of work as the two coaches could only deliver support to 


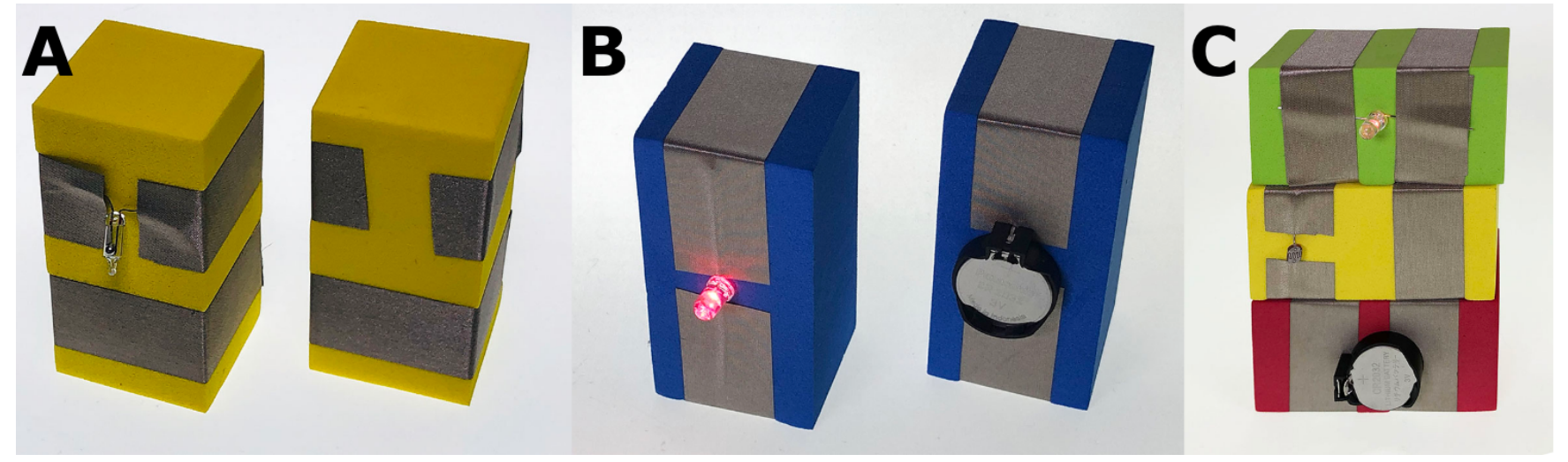

Figure 6: Samples of TapeBlocks. A) Front and back of tilt switch sensor block, B) Front and back of integrated block, C) TapeBlocks stack

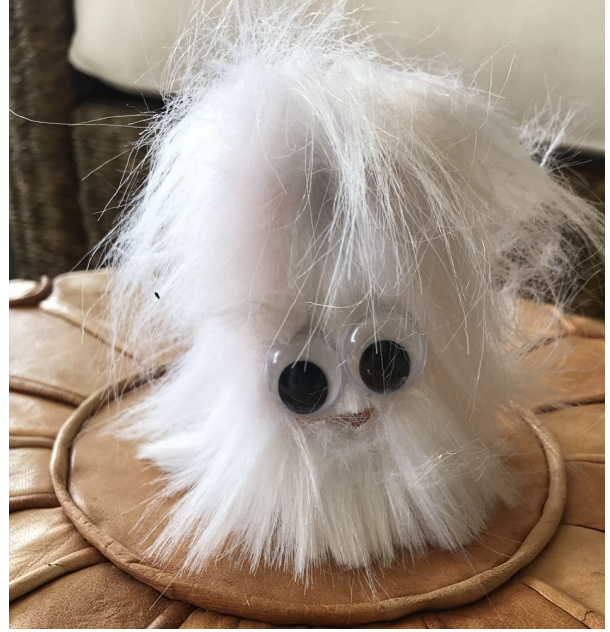

Figure 7: TapeBlocks character by a participant during maker workshops with white fur and googly eyes

one participant at a time. However, once again, the planned work was completed and everyone was successful in making their own personal creation.

\subsection{Post-Workshop Interviews}

After completing the four workshops, the young adults with intellectual disabilities and the support coaches took part in post-workshop interviews with a member of the research team. The interviews followed a semi-structured approach with a topic guide focused on the attitude towards the project pre- and post-engagement and improvements that could be made to the TapeBlocks, with the flexibility for other areas as needed. Each interview lasted for approximately 30 minutes. The young adults mentioned that they enjoyed their time in the TapeBlocks workshops and each of the four had different elements of the workshops that they had enjoyed most. Some preferred the earlier elements, like building vibrating or beeping blocks, and some enjoyed the more challenging activities in the final stages. All of the young adults noted that they had difficulty constructing the sensor based TapeBlocks because of the different pattern of tape on it. They also told the researcher they had used the pre-built TapeBlocks as references when they had become stuck. One of the participants expressed not liking their creation being wrapped in a furry material, because they did not enjoy the sensation of touching it. This suggested to us that having a range of materials to pick from, as most makerspaces do, would be beneficial.

The coaches were also happy with the TapeBlocks workshops and said they would feel confident running future workshops on their own. The coaches all wanted more material to support the TapeBlocks workshops but had diverging views on the specific nature of the materials. Some felt that the material needed to break down further, with a "plan B and plan C" for the slower and faster working clients, while others said that a single resource with a high degree of granularity would be sufficient. The coaches felt that the clients were engaged with TapeBlocks compared to other work and demonstrated solid retention of the ideas between workshops. Finally, the coaches expressed that delivering remote workshops posed significant challenges for them, despite knowing this prior to the workshops. At the same time, they felt that had they been running the workshops in-person, they would have been tempted to take control away from the young adults when they were struggling, which may not have been beneficial to the young adults' making experiences and learning process.

\section{DISCUSSION}

\subsection{Impacts of Online Delivery}

Changing the evaluation of TapeBlocks to work in an online environment showed us that our young adult participants were capable of consistently solving problems when left to work on them for long periods of time. The switch was challenging but it also highlighted a different set of characteristics of the toolkit than face-to-face workshops would have. The remote nature reduced the amount of 
support we could offer compared to traditional face-to-face workshops. Participants needed to work independently to solve problems, as we could not directly help participants complete an activity or troubleshoot. This led to a dynamic where finishing work was slower than we had anticipated, as coaches and researchers felt awkward at times that they could not assist a participant who had started to struggle.

However, every time this happened, participants were able to push through, complete their work, and expressed great satisfaction at doing so. We even saw evidence that participants were working out their own strategies to do this. Without requiring instructions, they found other blocks in their collection to use as references and started to develop debugging strategies such as checking for firm connections between blocks, rotations of the blocks or confirming the seating of batteries. Additionally, being forced to work through low resolution webcam images and erratic data connections made communication slower. In particular, it was hard to see what participants were doing as they could not point their laptop's inbuilt webcams at pictures while they were talking with us and, when they lifted up their work, orienting it so we could see the relevant sides of the work.

The issues of informed consent and safeguarding procedures around online delivery also needed to be taken into careful consideration. The research team closely worked with our NGO organisation to find solutions for recording workshops, administering ethical consent, and making sure that TapeBlocks were safely distributed.

\subsection{Revisiting TapeBlocks' Aspirations}

We consider the extent to which TapeBlocks has met the characteristics we set out for it in our design rationale.

Lowering the Threshold for Engagement. Across our workshops, we did not observe any hesitation from participants with wanting to start playing with the blocks, that can be seen with more complex starter kits. Some of our participants started to work with the kits we sent out and were able to get them working prior to the first workshop. Participants who had not played with the kit were able to make the TapeBlocks within the first 15 minutes in the first workshop without instructions. We suggest that one of the reasons for this is due to TapeBlocks' visibility and lack of hidden components. This facilitated the ability for participants to discover by doing. We observed them picking TapeBlocks up, feeling them, turning them over to look at the pattern of tape to gain an understanding of the connective nature of electronics components. By exploring and gaining direct results, participants were able to incrementally build an understanding from repeated self-facilitated experiments. This scaffolding of understanding of TapeBlocks also supported our meta-making activities when participants moved from using prebuilt TapeBlocks to form circuits to making their own blocks. This work was substantially slower than the pre-built blocks due to the required manual dexterity, the complexity involved in assembling the blocks correctly and finding the right components. However, the slower pace did not deter the participants from engaging with the process.

TapeBlocks' low threshold for engagement is also evident across the support coaches and carers. We were able to introduce TapeBlocks to support coaches in a single one-hour long workshop, after which they expressed confidence that they could run the maker workshops with minimal support from the research team. We did not closely consider the threshold for engagement for people who support those living with intellectual disabilities, as we did not expect direct participation. However, in our interviews with them post-workshop, they reported that the simplification of an electronics toolkit allowed them to step in and help participants when needed. They even reported enjoying playing with their TapeBlocks kits themselves and wanting to learn more about other making approaches.

Tinkerability. Throughout the workshops, participants were able to tinker with the blocks and sometimes use them for individual purposes. Participants made the TapeBlocks into tools to disrupt the class, using the beeper block, and played tunes with it. However, the exploration in this phase was more limited that we had envisioned when designing TapeBlocks due to the remote delivery. We did not have access to other tools what we could share with our participants to modify the TapeBlocks further.

Meaningful making and social character. We aimed to engender a sense of social connections with the TapeBlocks by letting people easily show their work to one another. In our evaluation, we saw evidence of the meaningful making and the social nature of TapeBlocks. Participants talked and offered each other help throughout the workshops, leveraging the oversized nature of the blocks to point to specific components of the blocks to aid their explanations. However, this was limited by the fact that only one person could talk at a time and the online platform made it difficult to show participants' progress.

The interplay between the coaches and the participants in the workshops mirrored parts of the classic maker environments but was more hierarchical. The coaches were adept at encouraging participants and constantly motivated them by showing faith in their ability to complete tasks without support. They nurtured participants' sense of curiosity by encouraging their exploration when they uncovered a new feature or function. Participants were challenged to race against each other to be the first to make something work. What is more, they were praised when they overcome more difficult obstacles complimenting their ability. This community is distinct from the more peer-driven ones in some makerspaces, but there were still signs of that camaraderie.

\subsection{What Comes After TapeBlocks?}

The onboarding process for makers is not typically straightforward. It requires learning multiple skills and deferring reward until they work through and gain a level of mastery in several of them. The TapeBlocks design pushes back against this, but it does not leave someone ready to start making on their own. Across the evaluation stages, all stakeholders - makers, coaches, and people living with intellectual disabilities - were interested in how to progress from TapeBlocks to more advanced making activities. We take this to be a positive sign, as the eagerness to progress and develop skills came from an underlying, newly found motivation to build more and engage with making.

Some participants were curious about how we could extend the TapeBlocks themselves as useful tools to build different things with 
them. Others were interested in customising the single block units in order to make them more expressive and bespoke. For makers, the idea seemed to tie into questions of support and granularity: while TapeBlocks highlights some problems with existing maker practices, completing the activities associated with TapeBlocks does not mean that participants are able to engage with makerspace tools on their own. The young adults living with intellectual disabilities wanted to know what new things they could build with the current toolkit and how they could expand it with new electronics components. One way to extend TapeBlocks would be to attach a Micro:bit with a banana plug and cut lead taped onto a TapeBlocks to facilitate programmable blocks and enable tinkering at a more advanced level. For support coaches, the extension focused on the new activities they could run and also made them think about how the TapeBlocks might work into an expanded repository of electronics and making activities.

\section{CONCLUSION}

In this paper we have presented TapeBlocks, a low cost, low fidelity toolkit that aims to improve the accessibility of making for people living with intellectual disabilities. We worked with makers and support coaches to evaluate the design of TapeBlocks and create a lesson structure that we then deployed with a group of young adults living with intellectual disabilities in remote workshop sessions. Our results showed that, despite the remote setting, participants were able to engage with the blocks to make their own electronics circuits and were able to build their own sets of blocks. We conclude that TapeBlocks supports accessible making and playful discovery of electronics for people living with disabilities, addressing a gap in existing toolkits by being tinkerable, affordable and having a low threshold for engagement.

\section{ACKNOWLEDGMENTS}

We wish to thank the teachers and coaches at Wallara for their generosity with their time and the makers who helped to evaluate the TapeBlocks concept.

\section{REFERENCES}

[1] Fraser Anderson, Tovi Grossman, and George Fitzmaurice. 2017. Trigger-ActionCircuits: Leveraging Generative Design to Enable Novices to Design and Build Circuitry. In Proceedings of the 30th Annual ACM Symposium on User Interface Software and Technology (Québec City, QC, Canada) (UIST '17). Association for Computing Machinery, New York, NY, USA, 331-342. https://doi.org/10.1145/ 3126594.3126637

[2] Giulia Barbareschi, Enrico Costanza, and Catherine Holloway. 2020. TIP-Toy: A Tactile, Open-Source Computational Toolkit to Support Learning across Visual Abilities. In The 22nd International ACM SIGACCESS Conference on Computers and Accessibility (Virtual Event, Greece) (ASSETS '20). Association for Computing Machinery, New York, NY, USA, Article 21, 14 pages. https://doi.org/10.1145/ 3373625.3417005

[3] Jeffrey Bardzell, Shaowen Bardzell, and Austin Toombs. 2014. "now That's Definitely a Proper Hack": Self-Made Tools in Hackerspaces. In Proceedings of the SIGCHI Conference on Human Factors in Computing Systems (Toronto, Ontario, Canada) (CHI '14). Association for Computing Machinery, New York, NY, USA, 473-476. https://doi.org/10.1145/2556288.2557221

[4] Ayah Bdeir. 2009. Electronics as Material: LittleBits. In Proceedings of the 3rd International Conference on Tangible and Embedded Interaction (Cambridge, United Kingdom) (TEI '09). Association for Computing Machinery, New York, NY, USA 397-400. https://doi.org/10.1145/1517664.1517743

[5] Cynthia L. Bennett, Abigale Stangl, Alexa F. Siu, and Joshua A. Miele. 2019 Making Nonvisually: Lessons from the Field. In The 21st International ACM SIGACCESS Conference on Computers and Accessibility (Pittsburgh, PA, USA)
(ASSETS '19). Association for Computing Machinery, New York, NY, USA, 279-285. https://doi.org/10.1145/3308561.3355619

[6] Paulo Blinkstein and Marcelo Worsley. 2016. Children are not Hackers - Building a Culture of Powerful Ideas, Deep Learning and Equity in the Maker Movement. In Makeology: Makerspace as Learning Enviroments, Kylie Peppler, Erica Rosenfeld Haverson, and Yasmin B. Kafai (Eds.). Vol. 1. Routledge, 64-81.

[7] Virginia Braun and Victoria Clarke. 2006. Using thematic analysis in psychology. Qualitative Research in Psychology 3, 2 (2006), 77-101. https://doi.org/10.1191/1478088706qp063oa https://www.tandfonline.com/doi/pdf/10.1191/1478088706qp063oa

[8] Craig Brown and Amy Hurst. 2012. VizTouch: Automatically Generated Tactile Visualizations of Coordinate Spaces. In Proceedings of the Sixth International Conference on Tangible, Embedded and Embodied Interaction (Kingston, Ontario, Canada) (TEI '12). Association for Computing Machinery, New York, NY, USA, 131-138. https://doi.org/10.1145/2148131.2148160

[9] Leah Buechley, Mike Eisenberg, Jaime Catchen, and Ali Crockett. 2008. The LilyPad Arduino: Using Computational Textiles to Investigate Engagement, Aesthetics, and Diversity in Computer Science Education. In Proceedings of the SIGCHI Conference on Human Factors in Computing Systems (Florence, Italy) (CHI '08). Association for Computing Machinery, New York, NY, USA, 423-432. https://doi.org/10.1145/1357054.1357123

[10] Erin Buehler, Niara Comrie, Megan Hofmann, Samantha McDonald, and Amy Hurst. 2016. Investigating the Implications of 3D Printing in Special Education. ACM Trans. Access. Comput. 8, 3, Article 11 (March 2016), 28 pages. https: //doi.org/10.1145/2870640

[11] Dorota Chapko, Pino Frumiento, Nalini Edwards, Lizzie Emeh, Donald Kennedy, David McNicholas, Michaela Overton, Mark Snead, Robyn Steward, Jenny M. Sutton, Evie Jeffreys, Catherine Long, Jess Croll-Knight, Ben Connors, Sam CastellWard, David Coke, Bethany McPeake, William Renel, Chris McGinley, Anna Remington, Dora Whittuck, John Kieffer, Sarah Ewans, Mark Williams, and Mick Grierson. 2020. "We Have Been Magnified for Years - Now You Are under the Microscope!": Co-Researchers with Learning Disabilities Created an Online Survey to Challenge Public Understanding of Learning Disabilities. In Proceedings of the 2020 CHI Conference on Human Factors in Computing Systems (Honolulu, HI, USA) (CHI '20). Association for Computing Machinery, New York, NY, USA, 1-17. https://doi.org/10.1145/3313831.3376278

[12] Australian Public Service Commission. 2017. Definition of disability. https://www.apsc.gov.au/definition-disability.

[13] Dale Dougherty. 2016. Free to make: How the maker movement is changing our schools, our jobs, and our minds. North Atlantic Books.

[14] Uta Frith and Christopher D. Frith. 1974. SPECIFIC MOTOR DISABILITIES IN DOWNS SYNDROME. Journal of Child Psychology and Psychiatry 15, 4 (1974), 293-301. https://doi.org/10.1111/j.1469-7610.1974.tb01253.x https://onlinelibrary.wiley.com/doi/pdf/10.1111/j.1469-7610.1974.tb01253.x

[15] Emilie Giles and Janet van der Linden. 2015. Imagining Future Technologies: ETextile Weaving Workshops with Blind and Visually Impaired People. In Proceedings of the 2015 ACM SIGCHI Conference on Creativity and Cognition (Glasgow, United Kingdom) (C\&C '15). Association for Computing Machinery, New York, NY, USA, 3-12. https://doi.org/10.1145/2757226.2757247

[16] Florian Güldenpfennig. 2017. Making a Customized DIY-Computer with Infobricks. (2017), 1-12. https://doi.org/10.14236/ewic/HCI2017.52

[17] Nic Hollinworth, Faustina Hwang, Kate Allen, Gosia Malgosia Kwiatkowska, and Andy Minnion. 2014. Making Electronics More Accessible to People with Learning Disabilities. In CHI '14 Extended Abstracts on Human Factors in Computing Systems (Toronto, Ontario, Canada) (CHI EA '14). Association for Computing Machinery, New York, NY, USA, 1255-1260. https://doi.org/10.1145/2559206.2581175

[18] Nicholas D. Hollinworth, Faustina Hwang, Kate Allen, Gosia Kwiatkowska, and Andy Minnion. 2014. LittleBits Go LARGE: Making Electronics More Accessible to People with Learning Disabilities. In Proceedings of the 16th International ACM SIGACCESS Conference on Computers \& Accessibility (Rochester, New York, USA) (ASSETS '14). Association for Computing Machinery, New York, NY, USA, 305-306. https://doi.org/10.1145/2661334.2661341

[19] Amy Hurst and Jasmine Tobias. 2011. Empowering Individuals with Do-ItYourself Assistive Technology. In The Proceedings of the 13th International ACM SIGACCESS Conference on Computers and Accessibility (Dundee, Scotland, UK) (ASSETS '11). Association for Computing Machinery, New York, NY, USA, 11-18. https://doi.org/10.1145/2049536.2049541

[20] Majeed Kazemitabaar, Liang He, Katie Wang, Chloe Aloimonos, Tony Cheng, and Jon E. Froehlich. 2016. ReWear: Early Explorations of a Modular Wearable Construction Kit for Young Children. In Proceedings of the 2016 CHI Conference Extended Abstracts on Human Factors in Computing Systems (San Jose, California, USA) (CHI EA '16). Association for Computing Machinery, New York, NY, USA, 2072-2080. https://doi.org/10.1145/2851581.2892525

[21] Ben Leduc-Mills, Jaymes Dec, and John Schimmel. 2013. Evaluating Accessibility in Fabrication Tools for Children. In Proceedings of the 12th International Conference on Interaction Design and Children (New York, New York, USA) (IDC '13). Association for Computing Machinery, New York, NY, USA, 617-620. https://doi.org/10.1145/2485760.2485882 
[22] Kevin Lefeuvre, Sören Totzauer, Andreas Bischof, Albrecht Kurze, Michael Storz, Lisa Ullmann, and Arne Berger. 2016. Loaded Dice: Exploring the Design Space of Connected Devices with Blind and Visually Impaired People. In Proceedings of the 9th Nordic Conference on Human-Computer Interaction (Gothenburg, Sweden) (NordiCHI '16). Association for Computing Machinery, New York, NY, USA, Article 31, 10 pages. https://doi.org/10.1145/2971485.2971524

[23] Jen Lewis. 2015. Barriers to women's involvement in hackspaces and makerspaces Access as spaces. Available at: http://access-space.org/wp-content/uploads/2015/10/ Barriers-to-womens-involvement-in-hackspaces-and-makerspaces.pdf (accessed 10 May 2016) (2015)

[24] MakeMedia. 2014. Attendee study Maker Faire Bay Area 2014. Retrived from http://make-cdn.s3.amazonaws.com/make/advertising/MFBA\%202014\% 20research\%20deck_FINAL.pdf.

[25] J. Maloney, L. Burd, Y. Kafai, N. Rusk, B. Silverman, and M. Resnick. 2004. Scratch a sneak preview [education]. In Proceedings. Second International Conference on Creating, Connecting and Collaborating through Computing, 2004, Kyoto, Japan, 104-109.

[26] Lauren R. Milne and Richard E. Ladner. 2018. Blocks4All: Overcoming Accessibility Barriers to Blocks Programming for Children with Visual Impairments. In Proceedings of the 2018 CHI Conference on Human Factors in Computing Systems (Montreal QC, Canada) (CHI '18). Association for Computing Machinery, New York, NY, USA, 1-10. https://doi.org/10.1145/3173574.3173643

[27] Vivian Genaro Motti and Anna Evmenova. 2019. Designing Technologies for Neurodiverse Users: Considerations from Research Practice.

[28] National Health Service. 2018. Severe head injury. https://www.nhs.uk/ conditions/severe-head-injury/

[29] Aisling Ann O'Kane, Amy Hurst, Gerrit Niezen, Nicolai Marquardt, Jon Bird, and Gregory Abowd. 2016. Advances in DIY Health and Wellbeing. In Proceedings of the 2016 CHI Conference Extended Abstracts on Human Factors in Computing
Systems - CHI EA '16. ACM Press, New York, New York, USA, 3453-3460. https: //doi.org/10.1145/2851581.2856467

[30] Jie Qi, Leah Buechley, Andrew "bunnie" Huang, Patricia Ng, Sean Cross, and Joseph A. Paradiso. 2018. Chibitronics in the Wild: Engaging New Communities in Creating Technology with Paper Electronics. In Proceedings of the 2018 CHI Conference on Human Factors in Computing Systems (Montreal QC, Canada) (CHI '18). Association for Computing Machinery, New York, NY, USA, Article 252, 11 pages. https://doi.org/10.1145/3173574.3173826

[31] Mitchel Resnick and Eric Rosenbaum. 2013. Designing for tinkerability. Design, make, play: Growing the next generation of STEM innovators (2013), 163-181.

[32] JooYoung Seo. 2019. Is the Maker Movement Inclusive of ANYONE?: Three Accessibility Considerations to Invite Blind Makers to the Making World. TechTrends 63, 5 (2019), 514-520. https://doi.org/10.1007/s11528-019-00377-3

[33] The Office Of National Statistics. 2019. Disability pay gaps in the UK: 2018. https://www.ons.gov.uk/peoplepopulationandcommunity/healthandsocialcare/ disability/articles/disabilitypaygapsintheuk/2018.

[34] Nick Taylor, Ursula Hurley, and Philip Connolly. 2016. Making Community: The Wider Role of Makerspaces in Public Life. In Proceedings of the 2016 CHI Conference on Human Factors in Computing Systems (San Jose, California, USA) (CHI '16). ACM, New York, NY, USA, 1415-1425. https://doi.org/10.1145/2858036.2858073

[35] Austin L. Toombs, Shaowen Bardzell, and Jeffrey Bardzell. 2015. The Proper Care and Feeding of Hackerspaces: Care Ethics and Cultures of Making. In Proceedings of the 33rd Annual ACM Conference on Human Factors in Computing Systems (Seoul, Republic of Korea) (CHI '15). Association for Computing Machinery, New York, NY, USA, 629-638. https://doi.org/10.1145/2702123.2702522

[36] Christopher James Vincent, Gerrit Niezen, Aisling Ann O'Kane, and Katarzyna Stawarz. 2015. Can Standards and Regulations Keep Up With Health Technology? FMIR mHealth uHealth 3, 2 (03 Jun 2015), e64. https://doi.org/10.2196/mhealth. 3918 